\title{
DIRECT METABOLIC ACTIVITY MEASUREMENT FOR UNSTABLE BIOPROCESS EXPERIMENT CONTROL
}

\author{
D. CHOINSKI \& P. SKUPIN \\ Faculty of Automatic Control, Electronics and Computer Science, Silesian University of Technology, Gliwice, Poland.
}

\begin{abstract}
Paper deals with an algorithm which allows the automatic selection of the best operating point of biological system. This task is one of the subjects of research in the field of metabolic engineering, which deals with control related issues, in particular, with modelling of biological phenomena, as well as, monitoring of the unstable states of biomass growth. The possibility of using specific biosensors and microfluidic system for monitoring, optimizing and controlling of a bioreactor is presented in this paper. To ensure proper experiment control of the bioreactor, a real-time measurement of parameters at the macroscale level and metabolic activity of microorganism cells at the microscale level are relevant. Therefore, much attention has been paid to the description and modelling of cyclical changes in metabolic states. For the determination of key process parameters, a microcalorimeter for measuring the heat of reaction has been applied. The biosensor provides additional information, which is useful in development of an interface for monitoring the bioreactor by a decomposition of measurements including the scale of process. Finally, the paper discusses the problem of model selection describing the bioprocess at the microscale level.

Keywords: Bifurcation analysis, control of experiment, metabolic flux analysis, microcalorimetry measurements, multiscale modelling, unstable bioprocess.
\end{abstract}

\section{INTRODUCTION}

Modern metabolic engineering is a discipline that uses the methods of many science and technology fields for the understanding and control of the processes belonging to the metabolic pathways. This is due to one of the most important tasks of the development of biotechnological process control, involving the integration of macroscopic analysis for the whole reactor with microscopic analysis of intracellular events. These reactions are mostly seen in the metabolic pathways catalyzed by enzymes. One method for the analysis of metabolic pathways is the method MFA (Metabolic Flux Analysis) [1,2]. This method is based on reducing the degrees of freedom of a biological system and from the observations of output metabolic fluxes of substrate(s) and product(s), it may be possible to determine the intracellular metabolic fluxes that are related to metabolic pathways.

The possibility of reducing the degrees of freedom of the biological system using external excitations offers ample opportunities to implement advanced control algorithms and control systems. Another effect of this reasoning is the ability to create sub-systems that isolate only part of the stream, associated with metabolic pathways (Varner and Ramkrishna [3]).

However, the described methodology is subject to the necessity of considering many phenomena simultaneously. Processes used in biotechnology have a high degree of the hierarchy (Shimizu [2]). The classic role of metabolic engineering is to increase the efficiency of the process in general, and from the point of view of individual cells-increasing the factor of growth productivity or the production of certain metabolites. These effects are usually the result of many cyclical changes and are not visible on a macroscopic scale, but the results of the different stages can be observed only at the micro level. For this reason, the number of variables necessary for observation and modelling, even for simple controls, is significant (Choinski et al., [4]).

Bacterial flora used in flow reactors is normally distributed freely as a so-called activated sludge. Current knowledge on the microbiology of activated sludge allows the description of the effect of 
biomass concentration on the rate of microbial metabolic reactions and balances of assimilated energy by microorganisms and used to sustain life processes and to carry out anabolic reactions that result in the formation of new biomass. Dependencies on the impact of the concentration on the distribution of biomass for the production of biomass energy and the efficiency of the process of metabolism are also known.

This knowledge, however, is currently not sufficiently used in the practical realizations of the wastewater treatment process control. Currently used algorithms for controlling plant biotechnology in most models use a steady state growth for the entire bioreactor.

\section{PRIMARY AND SECONDARY GROWTH OF MICROORGANISMS}

It is a generally described phenomenon that the cell accumulates a substrate in the form of accumulated PHB (polyhydroxybutyrate), or glycogen. For microbial sludge treatment plant biomass growth rate is relatively low and-as confirmed experimentally-accumulation is the predominant reaction (Sin et al. [5]). Numerous studies confirm that the observed changes in the transient behaviour of activated sludge are related to the changes in metabolic pathways corresponding to the primary and secondary growth phases. The primary growth phase - a large adsorption of substrate from the environment and simultaneous accumulation of its excess in cells. The secondary growth phase-utilization of the stored substrate (Karahan et al. [6]). These reactions can be characterized based on the behaviour of system at the microscale level, knowing that the dominant reactions are related to the accumulation of substrate and its utilization for further growth. The accumulation of substrate (an increase of $\mathrm{X}_{\mathrm{STO}}$ ) takes place when the excessive amounts of readily biodegradable substrate $\left(\mathrm{S}_{\text {in }}\right)$ provide adequate energy required for metabolic processes. In turn, the utilization of stored substrate (a decrease of $X_{\text {STO }}$ ) takes place during secondary growth and usually when the energy released from the breakdown of external substrate $\left(\mathrm{S}_{\text {raw }}\right)$ is not sufficient for the maintenance of metabolic processes or in the absence of substrate $\left(\mathrm{S}_{\text {in }}\right)$ inside a particular floc (see Fig.1).

At the same time it was assumed that, in the sustainable growth of biomass, the system producing proteins seeks to optimize the use of the substrate to achieve the required span of the population rather than achieve the maximum possible rate of growth (Grady et al. [7]). This assumption led to the development of mathematical models (Ni et al. [8]). These models primarily consider the interdependence of the concentration of extracellular polymers (EPS-extracellular polymeric substances including UAP-utilization-associated products and BAP-biomass-associated products); biomass accumulates inside the cell substrate $\left(\mathrm{X}_{\mathrm{STO}}\right)$ and products that are the result of the growth and decay of bacteria (SMP-soluble microbial products) for situation of repeating feast and famine conditions. Hence, it can be concluded that the kinetics of the unstructured models can be based on the description of unstable intracellular states provided that the phases of the metabolic pathways will be described as separate states. In the next sections we will describe a multi-scale analysis approach by making the distinction between macro- and microscale environments. Then, based on the given conditions, we will discuss the problem of model selection of the bioprocess and estimation of the key process parameters. The presented results are based on our earlier researches [4, 9-11].

\section{THE DISTINCTION OF MACRO- AND MICROSCALE ENVIRONMENTS}

Research at the microscopic level allows the preparation of mathematical models based on a matrix containing information about the kinetics of the individual reactions and the stoichiometric relationships. The values of the stoichiometric coefficients are determined in accordance with the law of conservation of mass, where the mass of the reactants is equal to the mass of the reaction products. 


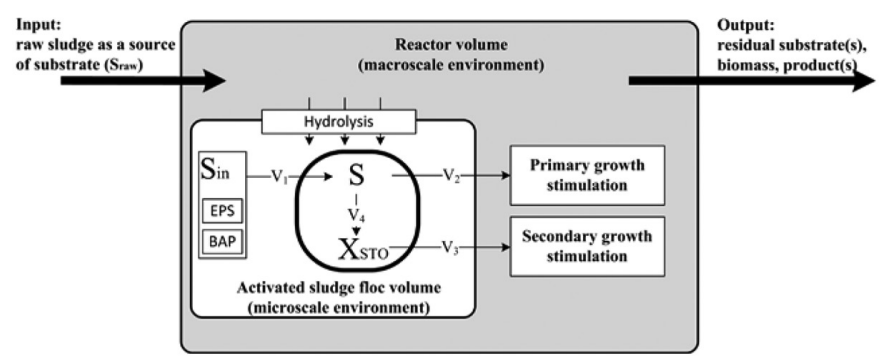

Figure 1: The idea of multi-scale analysis of the bioreactor fed with raw sludge containing dissolved organic matter $\left(\mathrm{S}_{\text {raw }}\right)$.

However, first, we have to make a distinction between macro- and microscale of the process. To gain an in-depth insight into the intracellular mechanisms (microscale), an excellent tool that can be used, is the MFA (van Impe et al. [12]). Figure 1 presents the possible structure of metabolic network considered, in our case, for single cells within the activated sludge floc volume. The substrate suspended in the bioreactor medium (macroscale environment) is transported into the cells (microscale environment) and then used for growth of the accumulated intracellular substrate. In the next phase (e.g. in the absence of readily biodegradable substrate), the accumulated intracellular substrate can be utilized for growth of the cell (Karahan et al. [6]).

By treating a single sludge floc as a well-stirred 'micro-bioreactor', which is fed with a single limiting substrate $\left(\mathrm{S}_{\mathrm{in}}\right)$, the governing equations of the system, corresponding to the metabolic network in Fig. 1, can be presented in a concise form by introducing the stoichiometric matrixes for external $S_{\text {ext }}$ and internal fluxes $S_{\text {int }}$, the vector of metabolic fluxes $v[\mathrm{~mol} / \mathrm{CFU} / \mathrm{h}]$ and the vector of intracellular components $\mathrm{C}[\mathrm{mol} / \mathrm{CFU}]$ :

$$
\frac{\mathrm{dC}}{\mathrm{dt}}=\left[\mathrm{S}_{\mathrm{ext}} \mid \mathrm{S}_{\mathrm{int}}\right] \cdot \mathrm{v}
$$

where:

$$
\mathrm{S}_{\mathrm{ext}}=\left[\begin{array}{ccc}
1 & -1 & 0 \\
0 & 0 & -1
\end{array}\right], \mathrm{S}_{\mathrm{int}}=\left[\begin{array}{c}
-\frac{1}{\mathrm{Y}} \\
1
\end{array}\right], \mathrm{v}^{\mathrm{T}}=\left[\mathrm{v}_{1}, \mathrm{v}_{2}, \mathrm{v}_{3}, \mathrm{v}_{4}\right], \mathrm{C}^{\mathrm{T}}=\left[\mathrm{S}, \mathrm{X}_{\mathrm{STO}}\right]
$$

$\mathrm{Y}$ is a stoichiometric coefficient, $\mathrm{S}$ is a substrate concentration [mol/CFU] inside the micro-bioreactor, which is not accumulated by the cell biomass, and $\mathrm{X}_{\mathrm{STO}}$ is the concentration of cell substrate [mol/CFU].

It should be noted that the yield coefficient determined for the single sludge floc can significantly vary depending on the changes in $\mathrm{S}$ and $\mathrm{X}_{\mathrm{STO}}$, while, at the same time, the yield coefficient determined for the whole bioreactor can be constant. This results from the obvious fact that the macroscale observations provide the information on the overall behaviour of bioreactor, which, in turn, results from the behaviour at the level of single cells (VanBriesen [13]). Hence, by focusing on the description of system at the microscale level, it will be possible to obtain information on how the individual 
intracellular fluxes affect the overall efficiency of bioprocess and how to stimulate the macroscale system to obtain the desired changes in metabolic fluxes.

\section{THE MEASUREMENT OF KEY PROCESS PARAMETERS}

A microcalorimeter is an excellent tool that can provide us with online information about the microscale process, i.e. for very small volumes. The device allows for measuring the heat of reaction on the order of several nW (Hurley et al. [14]). The prefix 'micro-' refers to the small volume of the sample (the sludge floc), which, in this case, is on the order of several microlitres. In effect, the sample has a very small heat capacity and the cost of experimentation is greatly reduced.

In order to measure the heat of reaction a microcalorimeter (NCM-9924) provided by the Xensor Integration can be used. The microcalorimeter chip has a thick silicon membrane, which is suited for liquids. The general scheme of the experimental set-up is shown in Fig. 2a. The integrated thermocouples allow for measuring the temperature difference, which arises between the centre of silicon membrane and the rim of device (in the reference temperature). Then, the temperature difference is converted to a voltage, which is proportional to the amount of heat evolved during the reaction process. To reduce the influence of ambient temperature, the microcalorimeter chip is covered with a PMMA block with two micro-channels (Fig. 2b).

The analysed sample is taken from the bioreactor and contains single flocs with some amount of the bioreactor medium, which, in turn, contains substrate of the concentration $\mathrm{S}_{\text {in }}$. This substrate can be used by the bacteria inside the floc. Hence, the amount of heat P[W] released by the reaction inside floc can be calculated from (Maskow et al. [15]):

$$
\mathrm{P}=\frac{\mathrm{D}\left(\mathrm{S}_{\text {in }}-\mathrm{S}\right)}{\mathrm{Y}} \Delta \mathrm{H}
$$

where: D-dilution rate $[1 / \mathrm{h}]$ defined as $\mathrm{D}=\mathrm{F} / \mathrm{V}$ (F-volumetric flow rate of the medium flowing through the sample, V-working volume of the sample), S-residual substrate concentration [mol/CFU], Y-yield coefficient [mol/mol], which is strongly dependent on the changes in $\mathrm{S}$ and $\mathrm{X}_{\mathrm{STO}}, \Delta \mathrm{H}$-the enthalpy of reaction $[\mathrm{kJ} / \mathrm{mol}]$. The changes in enthalpy are related to the chain reaction that can be affected by an appropriately chosen composition of substrate. However, the primary indicator of the dominant metabolic pathway (primary or secondary growth) is the concentration of cell substrate $\mathrm{X}_{\mathrm{STO}}$.

Because, the calorimetric measurements are carried out for small values of dilution rates $\mathrm{D}$, hence the substrate concentration in the output stream can be neglected as compared to $\mathrm{S}_{\text {in }}$ (Maskow et al.

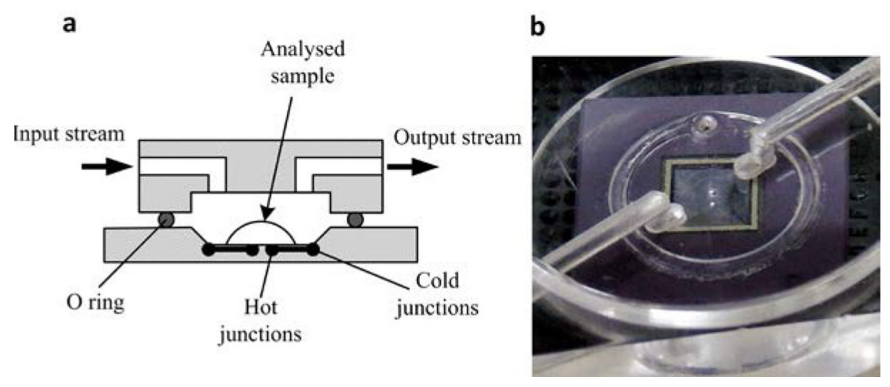

Figure 2: The experimental set-up. 
[15]). The concentration of substrate $\left(S_{i n}\right)$ suspended in the bioreactor medium, thus surrounding the floc containing the microorganism cells, can be easily measured and changed. The enthalpy of reaction $\Delta \mathrm{H}$ can be determined based on the calorimetric measurements of reaction heat $\mathrm{P}$ and elemental balance equations. The elemental composition of biomass, substrates or bacteria can be found in (see e.g. Grady et al.[16]) and the enthalpies for microbial biomass in (Cordier et al. [17]). As a result, the yield coefficient can also be calculated.

However, the main problem lies in the determination of dilution rate $\mathrm{D}=\mathrm{F} / \mathrm{V}$. The flow rate $\mathrm{F}$ can be measured by means of the specialized micro-flow meters or it can be assumed to be equal to the set flow rate of the syringe pump. In turn, determination of the working volume $\mathrm{V}$ can be quite difficult. Because, both the volume of sample and the flow rate $\mathrm{F}$ are rather very small, hence, the assumption of perfect mixing is not valid. Based on an appropriately chosen mathematical model it is possible to estimate the working volume V. From the energy conservation law the mathematical model describing the temperature changes of liquid flowing through the microcalorimeter chamber is as follows:

$$
\mathrm{V} \frac{\mathrm{dT}}{\mathrm{dt}}=\mathrm{F}\left(\mathrm{T}_{\mathrm{in}}-\mathrm{T}\right)+\frac{\mathrm{P}_{\mathrm{g}}}{\rho \mathrm{c}_{\mathrm{w}}}
$$

where: $\mathrm{T}_{\mathrm{in}}, \mathrm{T}$ - temperature of liquid in the input and output of calorimeter chamber, respectively, F-flow rate of liquid, which can be changed by means of the syringe pump, V-working volume of the calorimeter chamber, $\rho$-density of liquid, $c_{w}-$ specific heat of liquid, $\mathrm{P}_{\mathrm{g}}$-power of the heater, which is integrated in the calorimeter membrane. By using the model (4), it is possible to estimate the working volume $(\mathrm{V})$ for various liquids simply by fitting the response of device to the model.

In the next section, we will discuss a mathematical model of process at the microscale level that incorporates the changes in $\mathrm{X}_{\mathrm{STO}}$ and changes in the yield coefficient and its possible use for control purposes.

\section{MICROSCALE MODEL OF THE PROCESS}

In general, biological processes are difficult to model. This is due to the obvious fact that the biochemical reactions involving living microorganisms are very complex in nature. There are hundreds of intermediate reactions taking place in single cells. Hence, the description of all the intracellular reactions may be very difficult or even impossible. It means that we have to rely on the simplified models whose complexity depends on their structure, number of the intracellular components or metabolites. Mathematical models describing the kinetics of growth of microorganisms can be classified into two groups (Dunn et al. [18]), i.e. unstructured and structured models. In the unstructured models, the whole population of microorganisms is described by a single variable, i.e. biomass concentration. In this case, the bioreactor can be treated as a black box system with several inputs and several outputs (Nelson and Sidhu [19]). In turn, the structured models are more complex, because they include some intracellular processes and components.

In our case, the mathematical model must incorporate some intracellular mechanisms and components (e.g. cell substrate $\mathrm{X}_{\mathrm{STO}}$, which is accumulated inside the cells), which means that it must have some features specific for the structured models. On the other hand, the mathematical model should have a sufficiently simple structure to be well-suited for control purposes and this is specific for the unstructured models. Hence, the final form of the model equations is as follows:

$$
\frac{\mathrm{d} S}{\mathrm{dt}}=\mathrm{DS}_{\mathrm{in}}-\mathrm{DS}-\frac{1}{\mathrm{Y}} \frac{\mu_{\mathrm{m}} \mathrm{S}}{\mathrm{S}+\mathrm{K}_{\mathrm{s}}} \mathrm{X}_{\text {STO }}
$$




$$
\frac{\mathrm{dX}_{\mathrm{STO}}}{\mathrm{dt}}=-\mathrm{DX}_{\mathrm{STO}}+\frac{\mu_{\mathrm{m}} \mathrm{S}}{\mathrm{S}+\mathrm{K}_{\mathrm{s}}} \mathrm{X}_{\mathrm{STO}}
$$

where: $\mu_{\mathrm{m}}$ - maximum specific growth rate [1/h], $\mathrm{K}_{\mathrm{s}}$-half saturation constant [mol/CFU], the other parameters have been defined in the previous sections.

The presented model has the identical structure as in the case of the continuous flow bioreactor (chemostat) with the single limiting substrate (Nelson and Sidhu [19]). The presented analysis will be mainly based on numerical simulations of the model equations according to the algorithms presented in (Skupin [20]) and by numerical bifurcation analysis in continuation software XPPAut (Ermentrout [21]).

For the obvious reasons, our considerations will be limited to the set $\Omega=\left\{\left(\mathrm{S}, \mathrm{X}_{\mathrm{STO}}\right): \mathrm{S} \geq 0\right.$ and $\left.\mathrm{X}_{\mathrm{STO}} \geq 0\right\}$, which is positively invariant for the system (5)-(6) (Pilyugin and Waltman [22]). The equilibrium points of (5)-(6) can be found by setting all the derivatives equal to zero:

$$
\begin{gathered}
\mathrm{S}_{1}^{*}=\mathrm{S}_{\mathrm{in}}, \mathrm{X}_{\mathrm{STO} 1}^{*}=0 \\
\mathrm{~S}_{2}^{*}=\frac{\mathrm{DK}_{\mathrm{s}}}{\mu_{\mathrm{m}}-\mathrm{D}}, \mathrm{X}_{\mathrm{STO2}}^{*}=\mathrm{Y}\left(\mathrm{S}_{\mathrm{in}}-\frac{\mathrm{DK}_{\mathrm{s}}}{\mu_{\mathrm{m}}-\mathrm{D}}\right)
\end{gathered}
$$

The equilibrium points (7) and (8) correspond to washout and no washout states, respectively. It is well-known that the washout state can occur when the dilution rate is too high (Nelson and Sidhu [19]). According to van Impe and co-workers [12], this is unlikely situation, because the dilution terms (-DS or -DX) are often neglected as compared to the remaining fluxes. Therefore, we will focus our attention on the non-washout equilibrium point (8). The Jacobian matrix corresponding to system (5)-(6) at the point (8) has a form:

$$
J\left(S_{2}^{*}, X_{\text {STO2 }}^{*}\right)=\left[\begin{array}{cc}
-\mathrm{D}-\frac{\mu_{\mathrm{m}} \mathrm{K}_{\mathrm{s}} \mathrm{X}_{\mathrm{STO} 2}^{*}}{\mathrm{Y}\left(\mathrm{S}_{2}^{*}+\mathrm{K}_{\mathrm{s}}\right)^{2}} & -\frac{\mu_{\mathrm{m}} \mathrm{S}_{2}^{*}}{\mathrm{~S}_{2}^{*}+\mathrm{K}_{\mathrm{s}}} \cdot \frac{1}{\mathrm{Y}} \\
\frac{\mu_{\mathrm{m}} \mathrm{K}_{\mathrm{s}} \mathrm{X}_{\mathrm{STO} 2}^{*}}{\left(\mathrm{~S}_{2}^{*}+\mathrm{K}_{\mathrm{s}}\right)^{2}} & 0
\end{array}\right]
$$

and based on its Eigen values, the conditions for the local stability of (8) are:

$$
\operatorname{tr}(\mathrm{J})=-\mathrm{D}-\frac{\mu_{\mathrm{m}} \mathrm{K}_{\mathrm{s}} \mathrm{X}_{\mathrm{STO} 2}^{*}}{\mathrm{Y}\left(\mathrm{S}_{2}^{*}+\mathrm{K}_{\mathrm{s}}\right)^{2}}<0 \text { and } \operatorname{det}(\mathrm{J})=\frac{\mu_{\mathrm{m}}^{2} \mathrm{~K}_{\mathrm{s}} \mathrm{X}_{\mathrm{STO} 2}^{*} \mathrm{~S}_{2}^{*}}{\mathrm{Y}^{2}\left(\mathrm{~S}_{2}^{*}+\mathrm{K}_{\mathrm{s}}\right)^{3}}>0
$$

where $\operatorname{tr}(\mathrm{J})$ and $\operatorname{det}(\mathrm{J})$ are trace and determinant of the Jacobian matrix (9), respectively. The conditions (10) are satisfied, if $S_{2}^{*}>0$ (this is true for $D>0$ ) and $X_{\text {STO2 }}^{*}>0$, which is true if $S_{2}^{*}<S_{\text {in }}$ and this is guaranteed if $\mathrm{D}<\mathrm{D}_{\mathrm{c}}$, where: $\mathrm{D}_{\mathrm{c}}=\mu_{\mathrm{m}} \mathrm{S}_{\text {in }} /\left(\mathrm{S}_{\text {in }}+\mathrm{K}_{\mathrm{s}}\right)$. Hence, the final form of the stability condition of (8) is (Pilyugin and Waltman [22]):

$$
0<\mathrm{D}<\mathrm{D}_{\mathrm{c}}
$$

Further analysis has shown that the system described by the equations (5)-(6) does not exhibit oscillatory behaviour, if the yield coefficient $\mathrm{Y}$ is constant (see e.g. Huang [23]). 
It should be emphasized that the oscillatory changes of the cell substrate concentrations $\mathrm{X}_{\mathrm{STO}}$ are crucial in our considerations. Hence, in order to model the oscillatory behaviour, it is necessary to modify the structure of the model (5)-(6), but without increasing its complexity. We must still keep in mind that the mathematical model can be used in the model-based controller. This can be achieved by introducing a variable yield coefficient $Y$ to the model equations (see e.g. Huang [23]) in the following form:

$$
\mathrm{Y}(\mathrm{S})=\alpha+\beta S
$$

where: $\alpha, \beta$ - are constant and positive coefficients. By substituting the variable yield (12) to the equation (5), we receive a new form of the mathematical model that can have qualitatively different properties. Huang [23], Pilyugin and Waltman [22] have proved that if the yield coefficient $Y(S)$ is an increasing function of $S$ for $S>0$ and $\alpha, \beta>0$, then the system (5)-(6) exhibits oscillatory behaviour for some range of dilution rates $\mathrm{D}$.

A hypothesis concerning the dependence of the yield coefficient on the substrate concentration is well established in the literature and, in our case, it can be explained that the changes in the yield coefficient are related to the intracellular catabolic and anabolic processes. Moreover, the available substrate strongly affects the yield coefficient.

As previously mentioned, our considerations are still limited to the invariant set $\Omega=\left\{\left(\mathrm{S}, \mathrm{X}_{\mathrm{STO}}\right)\right.$ : $\mathrm{S}$ $\geq 0$ and $X_{\text {STO }} \geq 0$ \}. Again, by setting all the derivatives equal to zero (in the model (5) - (6) with variable yield coefficient) two equilibrium points can be found:

$$
\begin{gathered}
\mathrm{S}_{1}^{*}=\mathrm{S}_{\mathrm{in}}, \mathrm{X}_{\mathrm{STO} 1}^{*}=0 \\
\mathrm{~S}_{2}^{*}=\frac{\mathrm{DK}_{\mathrm{s}}}{\mu_{\mathrm{m}}-\mathrm{D}}, \mathrm{X}_{\mathrm{STO} 2}^{*}=\left(\alpha+\beta \mathrm{S}_{2}^{*}\right)\left(\mathrm{S}_{\mathrm{in}}-\mathrm{S}_{2}^{*}\right)
\end{gathered}
$$

As previously, the equilibrium points (13) and (14) refer to washout and no washout states, respectively. Hence, the further analysis will be limited to the second equilibrium point (14). The Jacobian matrix corresponding to system (5)-(6) with variable yield coefficient (12) at the point (14) has a form:

$$
J\left(S_{2}^{*}, X_{\text {STO2 }}^{*}\right)=\left[\begin{array}{cc}
-D-\frac{\mu^{\prime}\left(S_{2}^{*}\right)\left(\alpha+\beta S_{2}^{*}\right)-D \beta}{\left(\alpha+\beta S_{2}^{*}\right)^{2}} X_{\text {STO2 }}^{*} & -\frac{D}{\alpha+\alpha S_{2}^{*}} \\
\mu^{\prime}\left(S_{2}^{*}\right) X_{\mathrm{STO} 2}^{*} & 0
\end{array}\right]
$$

where: $\mu^{\prime}(\mathrm{S})$ is a derivative of $\mu(\mathrm{S})$ with respect to $\mathrm{S}$. Based on the Eigen values of (15), the conditions for the local stability of (13) are as follows:

$$
\mathrm{D}+\frac{\mu^{\prime}\left(\mathrm{S}_{2}^{*}\right)\left(\alpha+\beta \mathrm{S}_{2}^{*}\right)-\mathrm{D} \beta}{\left(\alpha+\beta \mathrm{S}_{2}^{*}\right)^{2}} \mathrm{X}_{\mathrm{STO} 2}^{*}>0, \text { and } \frac{\mu^{\prime}\left(\mathrm{S}_{2}^{*}\right) \mathrm{X}_{\mathrm{STO} 2}^{*} \mathrm{D}}{\alpha+\beta \mathrm{S}_{2}^{*}}>0
$$

The latter inequality is always satisfied for $D>0$, since the derivative $\mu^{\prime}\left(S_{2}^{*}\right)$ is always positive for $S_{2}^{*}$ $\geq 0$ and $\mathrm{X}_{\mathrm{STO} 2}^{*}>0$, if $\mathrm{D}<\mathrm{D}_{\mathrm{c}}=\mu_{\mathrm{m}} \mathrm{S}_{\text {in }} /\left(\mathrm{S}_{\mathrm{in}}+\mathrm{K}_{\mathrm{s}}\right)$. However, it can be shown that for some range of dilution rates $\mathrm{D}$ the first of the inequalities (16) is not satisfied and, as a result, the system (5)-(6) with variable yield coefficient (12) undergoes a Hopf bifurcation and the stable limit cycle occurs (Huang [23]). To examine the influence of $\alpha$ and $\beta$ parameters in eqn (12) on the existence of limit cycles, 

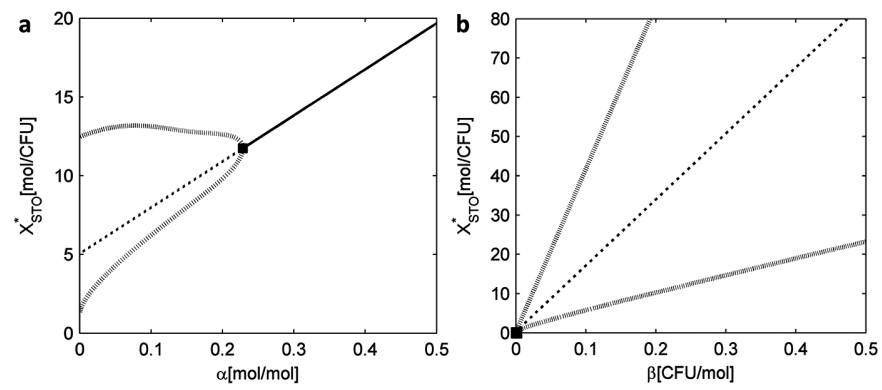

Figure 3: Steady state values of the biomass concentration $\mathrm{X}_{\mathrm{STO}}$ versus: a) $\alpha$ parameter; $b$ ) $\beta$ parameter. The continuous and broken lines represent stable and unstable branches, respectively. The black dot represents the point, for which $\mathrm{D}=\mathrm{D}_{\mathrm{c}}$, and the black filed rectangles represents Hopf points. The thick dashed lines represent the maximum and minimum values of $\mathrm{X}_{\mathrm{STO}}$ in the range of sustained oscillations. There is no oscillatory behaviour for $\beta=0$.

hundreds of simulation runs were carried out for various values of $\alpha$ and $\beta$ parameters and constant dilution rate. The obtained results were double checked in XPPAut and presented in Fig. 3. First, we examined the influence of $\alpha$ parameter by setting $\beta=$ const $=0.03[\mathrm{CFU} / \mathrm{mol}]$ (Fig. 3a), and then, the influence of $\beta$ for $\alpha=$ const $=0.01$ [mol $/ \mathrm{mol}$ ] (Fig. 3b). The oscillatory behaviour was observed for very small values of $\beta$. However, it should be emphasized that there were no oscillations for $\beta=0$ and when $\alpha$ parameter was much greater than $\beta$. In this cases the yield coefficient $Y$ was either constant (for $\beta=0$ ) or nearly constant (for $\alpha>>\beta$ ). It means that the strong variability of the yield coefficient is necessary to trigger the oscillations of $X_{\mathrm{STO}}$.

Now, in order to use the proposed mathematical model for control purposes, it is required to estimate its parameter values. However, due to its simplified structure and constant changes of the yield coefficient $\mathrm{Y}$, the estimation must be carried out online, according to the method described in Section 4.

\section{CONCLUDING REMARKS}

The proper stimulation of bioprocess requires the information about the intracellular fluxes and metabolic activity of the microorganisms. The individual intracellular fluxes affect the overall efficiency of bioprocess. The key issue in this paper was to make the distinction between the macro- and microscale levels of the bioprocess. This multi-scale approach allows for evaluation of the metabolic activity of microorganisms cells. This can be achieved by the application of microcalorimeter device. In turn, the proposed unstructured mathematical model gives the description of bioproces at the microscale level and can be used for control purposes. The presented researches and the future works are focused on the optimisation of unstable bioprocess.

\section{ACKNOWLEDGMENTS}

This work was supported by the National Science Centre under grant No. 2012/05/B/ST7/00096 and by the Ministry of Science and Higher Education under grant BK-UiUA.

\section{REFERENCES}

[1] Stephanopoulos, G. \& Vallino, J.J., Network Rigidity and Metabolic Engineering in Metabolite Overproduction. Science 252, pp. 1675-1681, 1991. doi: http://dx.doi.org/10.1126/science. 1904627 
[2] Shimizu, H., Metabolic Engineering-Integrating Methodologies of Molecular Breeding and Bioprocess System Engineering. Journal of Bioscience and Bioengineering, 94, pp. 563-573, 2002. doi: http://dx.doi.org/10.1263/jbb.94.563

[3] Varner, J. \& Ramkrishna, D., Metabolic Engineering from a Cybernetic Perspective.2. Qualitative Investigation of Nodal Architectures and Their Response to Genetic Perturbation. Biotechnology Progress, 15, pp. 426-438, 1999. doi: http://dx.doi.org/10.1021/bp990018h

[4] Choinski, D., Metzger, M., Nocon, W., Polakow, G. \& Skupin, P., AI-Based Support for Experimentation in an Environmental Biotechnological Process. Lecture Notes in Computer Science, eds. C. Sombattheera et al. Springer-Verlag Berlin Heidelberg, pp. 155-166, 2012. doi: http://dx.doi.org/10.1007/978-3-642-35455-7 15

[5] Sin, G., Gujsasola, A., de Pauw, D.J.W., Baeza, J.A., Carrera J. \& Vanrolleghem, P.A., A new Approach for Modeling Simultaneous Storage and Growth Processes for Activated Sludge Systems Under Aerobic Conditions. Biotechnology and Bioengineering, 92, pp. 600-613, 2005. doi: http://dx.doi.org/10.1002/bit.20741

[6] Karahan O., van Loosdrecht, M.C.M. \& Orhon, D., Modeling the Utilization of Starch by Activated Sludge for Simultaneous Substrate Storage and Microbial Growth. Biotechnology and Bioengineering, 94, pp. 43-53, 2006. doi: http://dx.doi.org/10.1002/bit.20793

[7] Grady, C.P.L., Smets B.F. \& Barbeau, D.S., Variability in kinetic parameter estimates: A review of possible causes and a proposed terminology. Water Research, 30, pp. 742-748, 1996. doi: http://dx.doi.org/10.1016/0043-1354(95)00199-9

[8] Ni, B.-J., Zeng, R.J., Fang, F., Xie, W.-M., Shen, G.-P. \& Yu, H.-Q., Fractionating soluble microbial products in the activated sludge process. Water Research, 44, pp. 2292-2302, 2010. doi: http://dx.doi.org/10.1016/j.watres.2009.12.025

[9] Choinski, D., Metzger, M., Nocon, W., Polaków, G., Rozalowska, B. \& Skupin, P., Cooperative Access to Hierarchical Data from Biotechnological Pilot-plant. Lecture Notes in Computer Science, ed. Y. Luo, Springer-Verlag Berlin Heidelberg, pp. 171-178, 2012. doi: http://dx.doi. org/10.1007/978-3-642-32609-7 24

[10] Skupin, P. \& Metzger, M., The Application of Multi-Agent System in Monitoring and Control of Nonlinear Bioprocesses. Lecture Notes in Computer Science, eds. E. Corchado et al., Springer-Verlag Berlin Heidelberg, pp. 25-36, 2012. doi: http://dx.doi.org/10.1007/978-3$\underline{642-28942-2 \quad 3}$

[11] Turek-Szytow, J., Choinski, D. \& Miksch, K., Properties of the activated sludge after lipase bioaugmentation. Enironment Protection Enigineering, 33, pp. 211-219, 2007.

[12] Van Impe, J.F., Vercammen, D. \& Van Derlinden E., Toward a next generation of predictive models: A systems biology primer. Food Control, 29, pp. 336-342, 2013. doi: http://dx.doi. org/10.1016/j.foodcont.2012.06.019

[13] VanBriesen, J.M. \& Evaluation of methods to predict bacterial yield using thermodynamics. Biodegradation, 13, pp. 171-190, 2002. doi: http://dx.doi.org/10.1023/a:1020887214879

[14] Hurley, K.D., Frederick, B.G, DeSistoa, W.J., van Heiningena, A.R.P. \& Wheelera, M.C., Catalytic reaction characterization using micromachined nanocalorimeters. Applied Catalysis A: General, 390, pp. 84-93, 2010. doi: http://dx.doi.org/10.1016/j.apcata.2010.09.035

[15] Maskow, T., Kemp, R., Buchholz, F., Schubert, T., Kiesel, B. \& Harms, H., What Heat is Telling Us about Microbial Conversions in Nature and Technology: From Chip- to Megacalorimetry. Microbial Biotechnology, 3, pp. 269-284, 2010. doi: http://dx.doi.org/10.1111/j.17517915.2009.00121.x

[16] Grady, C.P.L Jr., Daigger G.T. \& Lim H.C., Biological Wastewater Treatment. Martin Dekker Publishing: New York, pp. 70-71, 1999. 
[17] Cordier, J.L., Butsch, B.M., Birou, B. \& von Stockar, U., The relationship between elemental composition and heat of combustion of microbial biomass. Applied Microbiology and Biotechnology, 25, pp. 305-312, 1987. doi: http://dx.doi.org/10.1007/bf00252538

[18] Dunn, I., Heinzle, E., Ingham, J. \& Penosil, J., Biological Reaction Engineering :Dynamic Modelling Fundamentals with Simulation Examples. Wiley-VCH Verlag GmbH \& Co. KGaA: Weinheim, pp. 91-92, 2005. doi: http://dx.doi.org/10.1002/3527603050

[19] Nelson, M.I. \& Sidhu, H.S., Analysis of a chemostat model with variable yield coefficient. Journal of Mathematical Chemistry, 38, pp. 605-615, 2005. doi: http://dx.doi.org/10.1007/ s10910-005-6914-2

[20] Skupin, P., Simulation approach for detection of the self-sustained oscillations in continuous culture. Proc. of the 11th WSEAS Int. Conf. On Mathematics and Computers in Biology and Chemistry, eds. V. Monteanu, R. Raducanu, G., Dutica, A. Croitoru \& V.E. Balas, Iasi, pp. 80-85, 2010.

[21] Ermentrout, B., Simulating, analyzing, and animating dynamical systems. A guide to XPPAUT for researchers and students. SIAM series Software Environments Tools, 2002. doi: http:// dx.doi.org/10.1137/1.9780898718195

[22] Pilyugin, S.S. \& Waltman, P., Multiple limit cycles in the chemostat with variable yield. Mathematical Bioscience, 182, pp. 151-166, 2003. doi: http://dx.doi.org/10.1016/s00255564(02)00214-6

[23] Huang, X.C., Limit cycles in a continuous fermentation model. Journal of Mathematical Chemistry, 5, pp. 287-296, 1990. doi: http://dx.doi.org/10.1007/bf01166359 